\title{
Nuove prospettive storiografiche sulla Grande guerra: violenze, traumi, esperienze
}

Il testo propone, attraverso l'analisi delle recensioni pubblicate sulla rivista internazionale "First World War Studies", una rassegna su alcuni recenti studi storici sulla Prima guerra mondiale. Dopo la svolta degli anni Settanta-Ottanta, la storiografia sta continuando ad approfondire in particolare il tema dei risvolti sociali e culturali della Grande guerra.

The text proposes, by the analysis of the reviews published in the international journal "First World War Studies", a survey of some recent historical studies about the WWI. After the turn of the Seventies and Eighties, the historiography is continuing to explore in particular the theme of the social and cultural aspects of the Great War.

\section{Introduzione}

Da un certo punto di vista la Prima guerra mondiale può essere paragonata alla figura titanica e sublime di Friedrich Nietzsche. Se infatti, per gioco, volessimo cancellare di colpo la sua figura dalla storia della filosofia, improvvisamente non solo creeremmo un vuoto tematico notevole, ma non capiremmo più cos'è davvero il XX secolo. Le categorie di pensiero e i concetti chiave colti dal grande filosofo tedesco sono così radicati nell'analisi della realtà odierna, e probabilmente futura, che una loro improvvisa cancellazione comporterebbe un'immediata alienazione in chi tenta di relazionarsi profondamente con la realtà. Lo stesso può ben dirsi della Prima guerra mondiale. La storiografia in merito è quasi una- 
nimemente d'accordo nel riconoscere nella Grande guerra una cesura nella storia mondiale, l'evento che ha ribaltato le categorie mentali e materiali di gran parte del mondo. In questo essa è, di fatto, rivoluzionaria; un gigantesco salto nel buio, un capovolgimento completo dell'impianto concettuale della civiltà europea. David Fromkin [2005, 16] la definisce «l'evento più rilevante dell'era moderna». La prima e più ovvia conseguenza di tale rilevanza è una quantità smisurata di studi e ricerche sull' argomento, tanto che Martin Gilbert [1998, 657] sostiene che «il numero dei libri sulla prima guerra mondiale è talmente sterminato che nessun uomo potrebbe leggerli tutti nell'arco di una sola vita». Frase che viene confermata immediatamente da qualche dato statistico: per esempio Gilbert ci informa che Zara Steiner [1977], nella bibliografia del suo testo sulle origini del conflitto, Britain and the Origins of the First World War, elenca ben 335 opere riguardanti soltanto la politica britannica, oppure che Alan Palmer [1965], nel suo studio di 243 pagine sul fronte di Salonicco, The gardeners of Salonika, riporta in bibliografia 140 opere di carattere specialistico, per concludere sostenendo che «tentare di scrivere una storia della prima guerra mondiale in un unico volume equivale, dal punto di vista bibliografico, a tentare di scalare in una volta sola l'Everest, il Pelio e l'Ossa» [Gilbert 1998, 657].

Per qualsiasi studioso che intraprende una ricerca sulla Grande guerra il rischio di alienazione e disorientamento di fronte alla quantità smisurata di opere è sempre dietro l'angolo. Proprio per questo sono molto importanti studi che affrontano l'aspetto storiografico e bibliografico della Prima guerra mondiale e si soffermano soprattutto nel cercare di discernere le principali linee interpretative, per orientare lo studioso nell'impervio cammino della ricerca storica. Il presente testo intende offrire una panoramica, assolutamente non esaustiva, della recente storiografia sulla Grande guerra.

Ho diviso il lavoro in due parti: nella prima verranno inquadrate le storiche linee interpretative del conflitto, mentre nella seconda, molto più corposa, mi soffermerò sulle nuove e recenti tendenze della storiografia straniera, attraverso l'analisi dei testi pubblicati. In particolare mi soffermerò sulle recensioni dei testi, pubblicate negli ultimi tre anni dalla rivista semestrale "First World War Studies", edita dall'International Society for First World War Studies. Questa società, fondata a Lione nel settembre del 2001 da Jenny Macleod e Pierre Purseigle, vanta ormai la presenza di quasi 300 docenti e ricercatori provenienti da tutto il mondo. Con un'impostazione metodologica rigorosamente interdisciplinare ed esente da paradigmi nazionalisti, essa è volta a organizzare o patrocinare conferenze, seminari, workshop, pubblicazioni, al fine di promuovere lo studio della Prima guerra mondiale, di rafforzare la collaborazione internazionale, di incoraggiare la 
comparazione, di facilitare il dialogo tra i vari approcci di studio e tra le diverse generazioni di studiosi.

All'interno del sito della società è inoltre presente quella che probabilmente è la più aggiornata bibliografia (http://www.firstworldwarstudies.org/?page_id=875) oggi disponibile sulla Grande guerra, composta da una prima sezione dove sono presentate le recenti novità editoriali divise tra lingua inglese, francese e tedesca, seguita da una lista di bibliografie tematiche (fra le quali, di particolare interesse risulta la prima, Reference and Bibliography, che elenca i più importanti repertori bibliografici). L'intero albero bibliografico è consultabile anche attraverso Zotero ed è inoltre possibile rimanere sempre aggiornati sulle novità editoriali inserite nella bibliografia attraverso la mailing list e i social network Twitter e Facebook.

\section{Le linee interpretative tradizionali}

I primi tentativi di fornire chiavi di lettura sulla Prima guerra mondiale nacquero quasi con il conflitto stesso. Già nel famoso opuscolo del 1916, Imperialismo fase suprema del capitalismo, Lenin, aprendo la via dell'interpretazione marxista, sosteneva che la guerra fa parte della natura propria del capitalismo e che quindi il conflitto in corso fosse la diretta conseguenza della competizione tra stati capitalisti per la spartizione delle materie prime del pianeta. Analogamente, dall'altro lato, le potenze vincitrici generarono un punto di vista sulla Grande Guerra come conflitto tra la democrazia e l'autoritarismo, scaricando gran parte della responsabilità prima su Guglielmo II e quindi sulla Germania.

In seguito, verso la metà degli anni Venti, venne alla luce l'idea che la guerra fosse il risultato «di un manchevole sistema di relazioni internazionali. [Secondo questo punto di vista] era stata l'esistenza di un sistema di alleanze che dividevano l'Europa in due campi ad aver reso inevitabile la guerra» [Joll 1985, 5]. Per questo le vecchie diplomazie d'Europa vennero accusate di stringere accordi segreti in previsione del conflitto. A tali accuse, anche se non mirate, la Germania rispose rendendo noti, già negli anni Venti, i documenti diplomatici inerenti il periodo precedente al conflitto raccolti in una monumentale opera di trentanove volumi chiamati complessivamente Die Grosse Politik der Europäischen Kabinette: l'obiettivo di questa "messa a nudo" era quello di dimostrare che la diplomazia tedesca non si era comportata diversamente dalle altre europee. Ben presto 
la Germania venne seguita da altri paesi, come il Regno Unito a partire dal 1926 e la Francia dal 1930. Documenti che diedero vita a una seconda stagione di studi in cui storici eminenti di varie nazionalità - guidati dall'asserzione del grande storico tedesco Leopold von Ranke del Primat der Aussenpolitik, cioè il primato della politica estera nella determinazione di quella interna e nel destino generale dello stato - sottoposero l'intero quadro internazionale europeo al riesame.

La Seconda guerra mondiale aprì una successiva fase di studi, la terza, che scaturì dal bisogno di razionalizzare l'anomalia nazista all'interno della storia europea e soprattutto tedesca. In questo ambiente sorge il lavoro del 1961 di Fritz Fischer [1965], Griff nach der Weltmacht, giustamente considerato uno dei testi di riferimento sulle origini della Grande guerra. La famosa "controversia Fischer" che si scatenò dopo la pubblicazione del libro era basata su alcune tesi sostenute in esso: 1) il governo imperiale tedesco si era assunto il rischio di una guerra mondiale e, preventivamente a ciò, si era attivamente preparato a essa; 2 ) gli obiettivi annessionistici, tipici di una politica imperialista, erano sostenuti non solo da intransigenti pangermanisti come Ludendorff, ma anche da un vasto settore dell'opinione pubblica e da politici moderati raccolti attorno a Bethmann-Hollweg [Iggers 1981, 121]. Nel testo di Fischer non vi è, come lui stesso dice nella prefazione alla prima edizione tedesca, un atto d'accusa né un'apologia, ma un'operazione di raccolta dati e la loro presentazione secondo un quadro di causa-effetto: l'imperialismo tedesco sorse molto tardi «e allora, con la sua violenza dinamica e con la sua aspirazione morale a conquistarsi un "posto al sole", infastidì grandemente le altre potenze e così sconvolse l'Europa e il mondo intero» [Fischer 1965, XVI]. Fischer sosteneva inoltre la tesi, non meno importante e certo foriera di successivi studi, della continuità tra la politica bismarckiana e i due conflitti mondiali, legando la storia nazionale tedesca al nazismo e alimentando, di conseguenza, l'idea di una particolare e anomala "via tedesca alla modernità".

Un'altra grande e riconosciuta cesura storiografica nello studio del conflitto fu provocata dalle inquietudini della Guerra fredda e dall'analisi di quelle che sembravano delle analogie tra il presente e il primo quindicennio del Novecento. In particolare va ricordato il testo di Eric Hobsbawm [1987], The Age of Empire. 1875-1914, nel quale veniva sottolineato il peso determinante avuto dal complesso militare-industriale dei singoli paesi nello scatenamento del conflitto.

Gli anni Settanta-Ottanta segnarono anche l'inizio di una nuova stagione di studi, meno incentrata sulle cause ma più attenta ai processi, spesso di carattere culturale e mentale, sorti dalla guerra. I due nomi più significativi sono quelli di Paul Fussell [1984] ed Eric J. Leed [1985], storici statunitensi ai quali si devono rispettivamente The Great War and Modern Memory, del 1975, e No Man's 
Land. Combat \& Identity in World War I, del 1979. Nel primo testo, attraverso una serrata analisi di letteratura, poesia e memorialistica di guerra, si evidenziava che il conflitto mondiale era diventato paradigmatico per descrivere o analizzare quelli successivi: gli sconvolgimenti materiali e psichici avevano inciso talmente in profondità nella mentalità europea e mondiale da risultare non solo costitutivi della memoria moderna, ma anche sovvertitori dei tradizionali quadri mentali. In questo senso Fussell suggeriva che la cesura provocata dal conflitto 1914-18, oltre ad avere una natura geopolitica ed economica ${ }^{2}$, aveva anche e soprattutto una sostanza mentale e culturale.

Il secondo testo si muoveva sullo stesso piano di Fussell ma si incentrava maggiormente sul fenomeno della guerra di trincea e sul cambiamento che questo nuovo modus operandi aveva comportato per la mentalità individuale prima dei soldati e, poi, dei reduci tornati alla vita di tutti i giorni. Scompariva così l'ideale romantico del guerriero, della lotta marziale tra uomini, per lasciare il posto a una scenografia dove i veri attori protagonisti erano le nuove tecnologiche applicate agli armamenti, delle quali la regina assoluta risultava la mitragliatrice. In questo scenario gli uomini erano ridotti a merce e, proprio come nei saggi e romanzi di Ernst Jünger citati da Leed, gli uomini, come qualsiasi altro materiale mobilitato al fronte, finivano in questa «turbina alimentata dal sangue» che era la «terra di nessuno». In questo scenario il soldato viveva un'esperienza traumatica che ne alterava la personalità stessa. Leed sosteneva che le nevrosi, i miti e i riti creati dai soldati per spiegare l'inspiegabile che attorniava loro non rappresentavano una contingente regressione, ma una coerente conseguenza della società moderna e del tipo di guerra che essa soltanto aveva prodotto. Molto interessanti, in questo caso, sono gli apporti dati da altre discipline come l'antropologia, la sociologia e la psicologia, divenute assolutamente necessarie per spiegare certi avvenimenti del passato.

Gli anni Novanta hanno visto l'approfondimento di questa tematica e in particolar modo l'analisi delle conseguenze culturali, sociologiche e psicologiche del conflitto. Paradigmatico in questo ambito è il testo di Mosse [1990], Fallen Soldiers. Reshaping the Memory of the World Wars, in cui il grande storico tedesco, ebreo ed emigrato in America durante il regime nazista, analizzava l'elaborazione dell'esperienza di guerra da parte dei governi delle principali potenze antagoni- 
ste del conflitto: Germania, Francia, Inghilterra e Italia. Dall'analisi traspare la creazione di quello che Mosse ha chiamato «il mito dell'esperienza di guerra», cioè l'elaborazione di un immaginario collettivo sulla guerra che velava la realtà drammatica del conflitto: per esempio attraverso il tema del sacrificio, che trovava apogeo nella «mascolinità anonima» [Detti e Gozzini 2002, 27] del milite ignoto, con cui ai cittadini venne offerto un modello razionalizzante l'ecatombe dei figli e nel quale al tempo stesso la nazione acquistava un carattere sacrale e religioso, per il quale il sangue dei caduti appariva un giusto tributo.

Un ulteriore esempio della corrente di studi degli anni Novanta è quello dell'italiano Antonio Gibelli [1991] con il suo L'officina della guerra, in cui, attraverso il largo utilizzo di fonti psichiatriche e letteratura popolare, veniva indagata in chiave antropologica, sulla scia di Leed, la trasfigurazione mentale di chi partecipò al conflitto e le conseguenze della guerra sul breve e lungo periodo nel mondo mentale di soldati e veterani.

\section{Le nuove linee interpretative}

Esattamente come nel paragrafo precedente, in cui si è tentato di fornire lo scheletro essenziale delle interpretazioni storiografiche sulla Grande guerra, qui esporrò le recenti ed essenziali linee interpretative del conflitto che si evincono dall'analisi delle recensioni pubblicate dalla rivista "First World War Studies", prendendo come riferimento i volumi usciti negli ultimi tre anni. Una volta ottenuta la lista delle opere recensite (più o meno quaranta) e attraverso una comparazione delle medesime, ho cercato dei comuni denominatori che mi permettessero di raggruppare per aree tematiche o metodologiche $i$ testi più importanti e interessanti.

Naturalmente ci sono diverse opere che non rientrano in queste macroaree e, nello stesso tempo, la loro eterogeneità non mi ha permesso, o non ho ritenuto necessario farlo, di interpretarle sotto ulteriori macroprospettive. La motivazione di quest'ultima scelta è legata al fatto che si tratta di tematiche classiche nello studio della Grande guerra, come le biografie dei generali o la saggistica legata a tematiche militari, e che, nonostante la loro fondamentale importanza e il grande interesse, sono sempre state prodotte in grande quantità e probabilmente sempre lo saranno. Non rappresentano in questo una novità. $\mathrm{Ci}$ sono invece delle opere singole che mi è difficile racchiudere in uno schema unitario ma che sicuramente si distinguono per l'originalità del tema e delle fonti utilizzate. In particolar modo mi interessa sottolineare come gli studi negli ultimi anni hanno sempre più allargato il ventaglio di tematiche nell'analisi storiografica del conflitto: il trauma psicologico esperienziale della guerra, nei soldati e nei veterani; il tema della 
violenza, perpetrata soprattutto attraverso il lavoro forzato e i campi di prigionia; l'amministrazione dei territori occupati soprattutto dalla Germania e dall'Impero austro-ungarico; il tema degli esclusi, dove in particolar modo si parla di storia di genere e degli stranieri negli eserciti europei.

Inoltre, esaminando macroscopicamente le interpretazioni sulla Grande guerra, è possibile notare un fenomeno metodologico sotterraneo, quindi non immediatamente visibile, ma di grande rilievo: l'ampliamento della gamma di criteri, fonti e quindi discipline, utilizzati dagli studiosi per tentare di comprendere cause e conseguenze degli eventi del passato.

Se nel secolo scorso si era già affermata una storiografia interdisciplinare sulla Grande guerra, in questi ultimi anni il fenomeno è entrato in una fase di esponenziale aumento, tanto che la maggior parte delle opere qui recensite utilizza questa metodologia di studio. In alcuni casi la struttura del testo diventa essa stessa rivelatrice dell'impostazione metodologica multidisciplinare. Prendiamo il caso di A companion to World War I edito da John Horne [2010]: si tratta di un volume che comprende 38 saggi, tutti di autori diversi e suddivisi in cinque grandi sezioni (Origins; The Military Conflict; Faces of War; States, Nations, and Empires; Legacies). L'obiettivo del testo, come suggerisce lo stesso curatore, è quello di fornire al lettore tutta una serie di dialoghi tra diversi approcci allo studio della Prima guerra mondiale. Stesso discorso può essere fatto per la triplice curatela di Heather Jones, Jennifer O’ Brien, Christoph Schmidt-Supprian [2008], Untold war: New Perspectives in First World War Studies. Il testo nasce dalla raccolta dei papers presentati alla conferenza della Dublin International Society for First World War Studies e gli autori provengono da impostazioni metodologiche e disciplinari anche molto diverse. Nonostante in queste raccolte ci siano singoli saggi di notevole interesse, è chiaro che l'obiettivo di fondo di tali pubblicazioni non è fare il punto su un determinato tema, ma stimolare il dialogo tra materie che fino a non molto tempo fa erano considerate a compartimenti stagni.

Viene qui richiamata una non citata complessità, che rende la Grande guerra sostanzialmente un unicum negli studi storici. L'impossibilità, per lo studioso singolo, di afferrare aspetti complessi del conflitto e tradurli in una sintesi è determinata dalla grande quantità di discipline necessarie per approcciarsi alle diverse problematiche da affrontare. Una varietà di temi che un singolo studioso non può padroneggiare. Anche da queste banali riflessioni appare evidente quanto sia imprescindibile una metodologia multidisciplinare e di conseguenza un lavoro di sintesi che coinvolga molteplici studiosi. 


\section{Psicologia e medicina}

Passando a descrivere i risultati dell'analisi dei testi, una tendenza chiaramente identificabile della recente storiografia sulla Grande guerra è quella che segue la scia dei grandi maestri degli anni Settanta-Ottanta: va riconosciuta una certa attenzione all'analisi dell'esperienza del soldato in guerra attraverso l'utilizzo delle fonti di psicologia, psichiatria e medicina in generale. Inoltre esse rappresentano una sorgente di prim'ordine per studiare l'influenza della guerra nella società civile, sul breve, medio e lungo periodo. Alexander Watson [2009], per esempio, nel suo Enduring the great war: combat, morale and collapse in the German and British armies, ha analizzato e descritto i meccanismi di adattamento psicologico che permisero a soldati e ufficiali di non crollare nell'ambiente traumatico delle trincee. Dei meccanismi che, da una parte, permettevano di convincere se stessi di avere più probabilità di sopravvivenza nonostante l'evidenza del contrario e, dall'altra, hanno consentito a Watson di cogliere questo come fattore comune presente sia nella trincea tedesca sia in quella inglese. Un dato significativo quest'ultimo, considerando che la storiografia in merito ha sempre posto una sostanziale differenza tra l'esercito britannico e quello tedesco, figli di due società molto diverse, la prima paternalistica e la seconda fondamentalmente aristocratica. A questo comune denominatore psicologico Watson ha associato, in entrambi gli eserciti, il ruolo cardine svolto dai giovani ufficiali di prima linea nel mantenere alto il morale delle truppe.

Sempre nel 2009 è stato pubblicato il lavoro di Gregory Matthew Thomas, Treating the trauma of the Great War: soldiers, civilians, and psychiatry in France, 1914-1940, in cui vengono esaminati i traumi, e la conseguente insanità mentale che ne derivò, subiti dai soldati durante il conflitto, per poi descrivere le conseguenze che l'esperienza della guerra produsse nella società francese in tempo di pace. Significativo in questo senso è il fatto che molti pazienti studiati da Thomas sono donne o civili: descrivere le conseguenze traumatiche e psicologiche della guerra attraverso non solo i soldati ma anche chi non è stato in battaglia sottolinea la fortissima influenza che il conflitto ebbe nella società francese nel suo complesso. Conseguenze che Thomas ha registrato anche a livello della famiglia, colpita emotivamente ed economicamente per coprire i costi delle cure per malattie mentali. Per tentare di ovviare a questo drammatico problema, in Francia sorsero ospedali o semplici servizi open service che operavano al di fuori del tessuto ospedaliero psichiatrico in cui i pazienti, e i famigliari, potevano essere in parte alleviati dal carico finanziario in molti casi insopportabile.

Altri casi analoghi di studio sulla Grande guerra in questa medesima prospettiva 
di ricerca li troviamo per esempio in Before my helpless sight: Suffering, dying and military medicine on the Western Front, 1914-1918 dell'olandese Leo van Bergen [2009]. Il suo obiettivo è descrivere l'esperienza del ferito dal momento in cui riceveva i primi soccorsi sul campo di battaglia fino alla base ospedaliera, puntando l'attenzione non solo sui singoli passaggi medici, le prime cure, il trasporto, la prognosi, ma anche sull'esperienza emotiva del paziente e il grado di sofferenza che era obbligato a sopportare.

Anche a guerra conclusa i mutilati e feriti di guerra rimasero una questione delicata da trattare per i governi dei singoli paesi. Beth Linker [2011], nel suo War's waste: Rehabilitation in World War I, ha sostenuto che la riabilitazione divenne parte fondamentale di un programma di reinserimento del ferito di guerra all'interno della società. Tre erano le motivazioni di tale programma: primo, per ridurre il numero di war-damaged bodies che potrebbero agire negativamente sull'immaginario di guerra dei cittadini; secondo, per ridurre sensibilmente i costi della pensione di guerra altrimenti gravanti sulle spalle del governo; e terzo, per permettere al veterano di poter lavorare e quindi reinserirsi nella vita economica del paese.

Le conseguenze psicologiche del primo conflitto mondiale sono anche al centro del testo del francese Manon Pignot [2012], Allons enfants de la patrie: Génération Grande Guerre, in cui sono state studiate le ripercussioni emotive del conflitto nella "generazione della Grande guerra", cioè nelle persone nate nei primi anni del XX secolo e che non vissero la guerra da soldati, perché non ancora in età per partecipare alle operazioni militari. La domanda che l'autore si è posto è la seguente: come e quanto la Grande guerra risultò formativa per questa generazione? Pignot sostiene che questa generazione, una volta divenuta adulta, sviluppò uno straordinario obbligo morale di incarnare la memoria di chi, per il loro futuro, aveva combattuto e sofferto durante la guerra. Inoltre Pignot sostiene che non bisogna cadere nell'errore di ritenere l'esperienza di guerra vissuta dai bambini al singolare: bisogna distinguere nettamente il bambino che visse l'esperienza dell'occupazione dal bambino che invece ne rimase estraneo. Quest'ultimi non conobbero personalmente la paura che accompagnava l'avvicinamento e l'arrivo delle truppe tedesche, il suono dell'artiglieria, le risorse materiali delle famiglie distrutte e la completa trasformazione di qualsiasi elementare routine quotidiana. Nonostante ciò Pignot sostiene, attraverso l'analisi di disegni e progetti grafici, che il bambino che non subì l'occupazione militare non era certamente estraneo agli orrori della guerra e che, inconsciamente, la violenza delle trincee e la dura vita dei soldati entrarono nel suo universo simbolico. Nonostante ciò, l'autore sostiene che la divisione che intercorreva tra questi due gruppi di bambini era 
profonda proporzionalmente quanto quella tra i veterani e i civili. Interessante anche la tesi della trasformazione del concetto di paternità: durante la guerra, infatti, i padri obbligati dal conflitto a stare per lungo tempo lontano da casa erano ossessionati dalla paura che i figli potessero dimenticarli e così perdessero il tradizionale rispetto verso l'autorità paterna. Questo, secondo Pignot, generò un mutamento sensibile nell'identità paterna degli adulti reduci della Grande guerra. Last but not the least sono altri due casi che voglio porre all'attenzione dei lettori: The Great War and German Memory. Society, Politics and Psychological Trauma, 1914-1945, di Jason Crouthamel [2009], e Shell Shock Cinema: Weimar Culture and the Wounds of War, di Anton Kaes [2009]. Questi studi puntano a dimostrare che nella Germania del dopoguerra si sviluppò una tendenza conscia e inconscia a drammatizzare il tema del ritorno dalla guerra e i suoi protagonisti. Nel primo testo Crouthamel, attraverso il concetto di "war neurotic", ha inteso raccontare la lotta svolta dai veterani di guerra colpiti da nevrosi e malattie mentali contro la marginalizzazione a cui erano condannati sia nella Repubblica di Weimar sia soprattutto durante il Terzo Reich. I veterani nevrotici venivano trattati alternativamente o come vittime empatiche della guerra oppure come sintomi di una debolezza strutturale della società guglielmina. Nel secondo testo, Anton Kaes ha analizzato il tema della società traumatizzata attraverso la cinematografia tedesca avanguardista del dopoguerra. Presupposto fondamentale di tale analisi è riconoscere il cinema come analogo all'inconscio collettivo della società tedesca del tempo. Ad esempio, alla luce di ciò vengono analizzati il tema della partenza e ritorno della "generazione perduta" in Nosferatu di F.W. Murnau del 1922, oppure la ricerca di un passato mitologico da abbinare all'apocalittico presente in Die Nibelungen di Fritz Lang del 1924, o ancora, la critica alla modernità industriale di massa in Metropolis, sempre di Lang, del 1927.

L'accettazione dell'interpretazione psicoanalitica delle fonti è essenziale soprattutto per una nuova lettura delle stesse. Paradigmatici in questo caso sono i disegni di bambini in Pignot e la cinematografia in Kaes. In particolare bisogna riconoscere che dietro il disegno di un bambino c'è più di quello che l'occhio immediatamente percepisce, o che il cinema può essere colto come inconscio collettivo di un'intera nazione. Ma si tratta di scogli metodologici ancora da definire e superare. 


\section{Una guerra non solo militare}

La Grande guerra può essere considerata la prima guerra totale. Già nel 1930 Ernst Jünger, in un piccolo saggio dal titolo Die Totale Mobilmachung, individuò l'unicità storica del conflitto nella "mobilitazione totale", ovvero nella disponibilità degli stati impegnati nel conflitto a dispiegare non solo la loro potenza militare e politica, ma anche e soprattutto quella sociale. Questo significa che lo stato impegnava la nazione ad adottare un programma di organizzazione industriale del lavoro su larga scala, prima per il riarmo, poi successivamente per il mantenimento e, se possibile, l'accrescimento dei rifornimenti al fronte. Ecco che quindi il nuovo sforzo bellico imposto dalla mobilitazione totale necessitava non solo della solida gestione di un esercito che combattesse il nemico al fronte, come succedeva per le guerre precedenti, ma anche che l'intero apparato produttivo nazionale su vasta scala, dall'agricoltura fino all'industria pesante, si coordinasse per garantire un costante afflusso di rifornimenti. Questo ha imposto un coinvolgimento completo di tutta la società nel conflitto. La risposta della storiografia a questo dato di fatto è quella di un allargamento delle tematiche di ricerca che coinvolgano ogni aspetto della guerra, vista questa volta da dietro il fronte, nelle città, nelle campagne o nella vita quotidiana. Il risultato è un mix di studi su argomenti anche molto differenti gli uni dagli altri, in cui la guerra non è più militare, ma sociale. In questa scia si collocano lavori trasversali al tema del conflitto armato, che arricchiscono le conoscenze sui contesti e gli ambienti in cui esso si svolse.

Questo aspetto della storiografia sul primo conflitto mondiale viene posta in evidenza, per esempio, da Tammy M. Proctor [2010]: in Civilians in a world at war, 1914-1918, egli si è interrogato sul concetto di combattente e ha messo in evidenza come questa parola, durante la guerra, non implicava più una distinzione netta tra militari e civili. Una problematica, questa, già implicita nel concetto di mobilitazione totale: in questo senso va intesa la locuzione Home Front, per cui al cittadino si chiedeva, nonostante i bombardamenti aerei, la carenza alimentare e le restrizioni di guerra, di conservare un autocontrollo e una disciplina indispensabili per assicurare l'essenziale supporto ai soldati al fronte.

La guerra del passato, cioè lo scontro tra eserciti di due o più contendenti, con la Prima guerra mondiale scomparve per lasciare il posto alla guerra tra nazioni, in cui l'esercito aveva la funzione di avanguardia, ma che dietro di sé contava su un complesso sistema industriale, amministrativo, politico, volto al mantenimento e, possibilmente, all'incremento della potenza bellica. Tuttavia, va sottolineato che, dietro le istituzioni amministrative, politiche ed economiche, c'era sempre l'uo- 
mo e la sua vita. I testi successivi si segnalano per aver utilizzato un approccio "dal basso".

Prendiamo il caso del testo di Xu Guoqi [2011], con il suo Strangers on the Western Front: Chinese laborers in the Great War, in cui viene analizzato il caso dei lavoratori cinesi emigrati in Europa tra l'estate del 1916 e la fine del conflitto e impiegati dietro le linee francesi e inglesi in lavori di ogni tipo. In questo intervallo di tempo, circa 150.000 cinesi arrivarono in Europa. Di questi, più o meno 100.000 lavorarono per gli inglesi e vennero organizzati nel Chinese Labour Corps $(\mathrm{Clc})$, mentre i restanti 50.000 vennero impiegati dietro le linee francesi. La tesi di Guoqi è duplice: da una parte sostiene che il processo di reclutamento, l'organizzazione e l'esperienza dei lavoratori cinesi sono state centrali per lo sviluppo di una nuova identità nazionale e per l'internazionalizzazione della Cina nella prima metà del XX secolo; e, dall'altra, sostiene che il contributo dato dal lavoro dei cinesi emigrati, per nulla studiato, è stato invece considerevole per l'esito finale del conflitto.

L'attenzione all'aspetto sociale della guerra, già evidente nel testo di Guoqi, si palesa anche nel contributo di David Laskin [2010] in The long way home: An American journey from Ellis Island to the Great War. Il testo si concentra sulla vita di 12 uomini che combatterono nell'esercito americano, 11 dei quali nati in paesi stranieri (Italia, Polonia, Norvegia, Russia, Ucraina, ecc.), il rimanente figlio di immigrati in America. Laskin sostiene che seguendo la loro storia è possibile cogliere sfumature altrimenti difficili da afferrare. Per esempio l'interazione tra loro e i nativi americani durante le esercitazioni militari e i comuni rischi sul campo di battaglia fecero sorgere, e poi rafforzarono, il loro senso di appartenenza alla comunità americana. Ciò favorì inoltre, da parte dei soldati americani, la percezione degli immigrati come compatrioti. Una delle tesi di fondo del testo è dunque che l'addestramento fornito dall'esercito rappresentò un passaggio fondamentale per diventare americani.

Altro tema molto importante emerso in queste analisi è quello della violenza dietro la linea del fronte, declinata in molteplici modalità: prigionia di guerra, lavoro forzato, occupazione armata.

Per quanto riguarda la prima Heather Jones [2011], in Violence against prisoners of war in the First World War, si è concentrata sul tema del prigioniero di guerra, sottolineando che il livello di brutalizzazione a cui i prigionieri venivano sottoposti in Germania, Francia e Inghilterra segnò uno spartiacque nell'evoluzione dell'idea di "lavoro forzato" nell'Europa occidentale. Ponendo l'inizio di questa escalation di violenza nel conflitto stesso, Jones ha analizzato il rapporto che intercorreva tra violenza e società, e nello stesso tempo ha dimostrato che il 
periodo di guerra vide sorgere, nei paesi sopracitati, come causa e conseguenza insieme, una cultura dell'odio radicata. In particolare l'autrice si è concentrata sulla complessa e simbiotica relazione tra violenza praticata e violenza immaginata. Nella sua analisi dei singoli stati è risultato come fosse la Germania il paese in cui venne raggiunto il maggior livello di violenza, in parte a causa della struttura dell'esercito e dell'amministrazione del personale carcerario. Per esempio, Jones ha evidenziato che, a differenza di Francia o Gran Bretagna, in Germania le guardie erano direttamente responsabili dei prigionieri, e ciò determinava comportamenti più violenti, per paura che i prigionieri scappassero, o per il bisogno di mantenere la disciplina ecc..

Anche Christian Westerhoff [2012], in Zwangsarbeit im Ersten Weltkrieg. Deutsche Arbeitskräftepolitik im besetzten Polen und Litauen 1914-1918, si è concentrato sul concetto di "lavoro forzato" e ne ha analizzato le molteplici sfumature, mettendo in luce per esempio che nella Germania dell'epoca il termine copriva diversi significati, tra cui corvée per costruire strade, la proibizione per i lavoratori stranieri di tornare nella loro patria e l'utilizzo di veri e propri battaglioni di lavoro. Fin dall'inizio della guerra, e specialmente dopo il 1915, la Germania accusò la mancanza di forza lavoro da impiegare nelle proprie fabbriche. La risposta a questo problema, sostiene Westerhoff, fu duplice: in primo luogo, la negazione di rimpatrio a chi era immigrato in Germania, una decina d'anni prima, per lavorare temporaneamente la terra, tra i quali la maggior parte polacchi delle province appartenenti all'Impero russo; in secondo luogo, dopo lo scoppio della guerra, il reclutamento di forza lavoro nei territori occupati dall'esercito tedesco, in particolare in Belgio e Polonia. Molto significative sono le differenze riscontrate da Westerhoff nel trattamento di questi territori: non solo, sostiene l'autore, vi fu differenza di comportamenti tra Est ed Ovest, ma nel caso polacco si può notare un'abissale disparità tra i lavoratori dei territori gestiti direttamente dall'Ober Ost (Oberbefehlshaber der gesamten Deutschen Streitkräfte im Osten, Comando supremo di tutte le forze tedesche nell'Est), generalmente trattati peggio, e quelli non ricaduti sotto di esso.

L'altra declinazione del tema della violenza, durante l'occupazione militare, è affrontata in almeno tre testi: The resurrection and collapse of Empire in Habsburg Serbia, 1914-1918 di Jonathan E. Gumz [2009]; Les cicatrices rouges, 14-18: France et Belgique occupées di Annette Becker [2010]; Zwischen Freund un Feind - Deutsche Besatzung in Rumänien 1916-1918, di Lisa Mayerhofer [2010]. Nel primo testo, l'obiettivo principale di Gumz è valutare come il tipo di occupazione asburgica della Serbia abbia rappresentato un continuum in linea politica e pratica fin dal periodo napoleonico, passando per l'esperienza della colonizzazio- 
ne europea, fino a presagire le pratiche totalitarie della Seconda guerra mondiale. Annette Becker, invece, ha riportato alla luce la storia dimenticata e mai studiata di coloro che, francesi o belgi, durante la lunga ed estenuante guerra di logoramento sul fronte occidentale, si trovavano sotto l'occupazione tedesca. L'autrice racconta attraverso i ritratti di alcune vittime le continue violazioni della Convenzione dell'Aia del 1899 e del 1907 compiute dall'esercito tedesco a danno della popolazione civile. Il terzo testo, invece, è uno dei pochi casi di studi sul fronte balcanico e analizza il tema dell'occupazione tedesca della Romania, sostenendo che essa rappresentò un unicum rispetto alle altre occupazioni militari durante il conflitto. Il motivo di tale differenza sta nel fatto che l'élite rumena cooperò con l'esercito e l'amministrazione tedesca nella gestione del territorio. Questo processo fu facilitato dal fatto che la dinastia rumena aveva un legame molto stretto con il Reich tedesco ed esisteva una forte fazione pangermanica nel Partito conservatore rumeno.

Infine, un altro filone di ricerca interessante è legato al tema dell'attivismo femminile nella Grande guerra e trova espressione nel testo di Katherine Storr [2010], Excluded from the record: Women, refugees and relief, 1914-1929. L'autrice si pone in forte polemica con gli studi tradizionali, considerati "patriarcali". La Storr sostiene che il pregiudizio della tradizionale storiografia nei confronti delle donne non abbia permesso di mettere in evidenza il grande contributo da esse offerto nel mitigare l'orrore della guerra: per esempio, il ruolo svolto dalle donne inglesi nell'accoglienza dei rifugiati dal Belgio o nell'assistenza alle famiglie di origine tedesca che vivevano in Gran Bretagna. La storiografia inoltre pare non abbia messo mai messo a fuoco il fatto che se per i soldati la guerra si concluse nel 1918, non fu così per alcune donne che, a lungo, dopo l'armistizio, continuarono a lavorare e lottare per salvare vite, rimpatriare persone disperse e svolgere attivismo.

Altro esempio di questo filone di ricerca è The search for negotiated peace: women's activism and citizen diplomacy in World War I di David Patterson [2008]. In questo testo l'autore ha affrontato il tema dell'impegno come mediatori di pace svolto da cittadini, in particolare donne, prima che gli Stati Uniti entrassero nel conflitto. Questa attività venne esercitata attraverso l'organizzazione di conferenze, l'incontro con i leader dei paesi in guerra, o il tentativo di convincere il Presidente Woodrow Wilson a portare i belligeranti sul tavolo della pace. Inoltre il testo, in sintonia con quello precedente della Storr, aiuta a comprendere la centralità del ruolo della donna nelle relazioni con gli stranieri e gli emarginati. Quello che si evince è l'importante ruolo giocato dalle persone comuni nel determinare le dinamiche quotidiane di eventi di grande portata storica. 


\section{Conclusioni}

Dall'analisi delle recensioni pubblicate in "First World War Studies" si apprende che la storiografia sulla Grande guerra sta seguendo ancora principalmente la direttrice di marcia impressa dagli studi pionieristici degli anni Settanta. Nonostante non manchino saggi su temi "classici", gli storici, piuttosto che soffermarsi senza fine sulle cause (e quindi, indirettamente, le colpe) del conflitto, preferiscono volgere lo sguardo verso l'esperienza della guerra e le conseguenze di lungo periodo che essa ha comportato per le società in tempo di pace. Questo ha portato a evidenziare l'uomo in guerra, soldato o civile che sia, non solo come attore in campo bellico, ma anche come soggetto psicologico dotato di una propria sfera emotiva.

In genere ogni aspetto del passato presenta particolari complessità che ne impongono la lettura multidisciplinare, ma la Prima guerra mondiale rappresenta in questo senso un unicum sorprendente che impone un decisivo ripensamento su quali siano le famose "ancelle della storia". Senza prendere in considerazione il tema delle cause o delle origini, che probabilmente, come un Joll rassegnato ha suggerito citando Wagner nell'incipit del suo lavoro del 1984, finirebbe per deviare verso la metafisica, lo studio dell'esperienza di guerra non può più prescindere dal contributo di discipline come la psicologia, la sociologia, la medicina. La complessità dell'evento non si attenua con il "cessate il fuoco", ma anzi, con il sopraggiungere del dopoguerra e la valutazione delle conseguenze sul breve e lungo periodo, la trama diventa ancora più ardita e complicata. Proprio per questo appare evidente come la storia della Grande guerra non possa essere davvero sviscerata dallo sforzo di un solo studioso che ne propone la sintesi, ma necessiti della collaborazione di un gruppo di ricercatori che lavori in stretto rapporto. Non stupisce, a questo punto, che la bibliografia offerta dall'International Society for First World War Studies si chiami, appunto, Collaborative Bibliography.

\section{Bibliografia}

Barraclough G. 1971, Guida alla storia contemporanea, Roma-Bari: Laterza (ed. or. 1964).

Becker A. 2010, Les cicatrices rouges, 14-18: France et Belgique occupées, Paris: Fayard.

Crouthamel J. 2009, The Great War and German memory. Society, politics and psychological trauma, 1914-1945, Exeter: University of Exeter Press.

Detti T. e Gozzini G. 2002, Storia contemporanea, II, Il Novecento, Milano: Bruno Mondadori. 
Fischer F. 1965, Assalto al potere mondiale. La Germania nella guerra 1914-1918, Torino: Einaudi (ed. or. 1961).

Fromkin D. 2005, L'ultima estate dell'Europa. Il grande enigma del 1914: perché è scoppiata la prima guerra mondiale?, Milano: Garzanti (ed. or. 2005).

Fussell P. 1984, La Grande Guerra e la memoria moderna, Bologna: il Mulino (ed. or. 1975).

Gibelli A. 1991, L'officina della guerra. La Grande Guerra e le trasformazioni del mondo mentale, Torino: Bollati Boringhieri.

Gilbert M. 1998, La grande storia della prima guerra mondiale, Milano: Arnoldo Mondadori (ed. or. 1995).

Gumz E.J. 2009, The resurrection and collapse of Empire in Habsburg Serbia, 1914, 1918, New York: Cambridge University Press.

Guoqi X. 2011, Strangers on the Western Front: Chinese laborers in the Great War, Cambridge: Harvard University Press.

Hobsbawm E.J. 1987, L'età degli imperi 1875-1914, Milano: Arnoldo Mondadori (ed. or. 1987).

Horne J. (ed.) 2010, A companion to World War I, Chichester: Wiley - Blackwell.

Iggers G.G. 1981, Nuove tendenze della storiografia contemporanea, Catania: Edizioni Del Prisma (ed. or. 1975).

Joll J. 1985, Le origini della prima guerra mondiale, Roma-Bari: Laterza (ed. or.1984).

Jones H. 2011, Violence against prisoners of war in the First World War, Cambridge: Cambridge University Press.

Jones H., O’ Brien J. e Schmidt-Supprian C. (eds.) 2008, Untold war: New perspectives in First Worlds War studies, Lieden-Boston: Brill.

Kaes A. 2009, Shell shock cinema: Weimar culture and the wounds of war, PrincetonOxford: Princeton University Press.

Laskin D. 2010, The long way home: An American journey from Ellis Island to the Great War, New York: Harper Collins.

Leed E.J. 1985, Terra di nessuno. Esperienza bellica e identità personale nella prima guerra mondiale, Bologna: il Mulino (ed. or. 1979).

Linker B. 2011, War's waste: Rehabilitation in World War I America, Chicago: University of Chicago Press.

Mayerhofer L. 2010, Zwischen Freund und Feind - Deutsche Besatzung in Rumänien 1916-1918, München: Meidenbauer.

Mosse G.L. 1990, Le guerre mondiali. Dalla tragedia al mito dei caduti, Roma-Bari: Laterza (ed. or. 1990).

Palmer A. 1965, The gardeners of Salonika, London: Deutsch.

Patterson D. 2008, The search for negotiated peace: Women's activism and citizen diplo- 
macy in World War I, New York: Routledge.

Pignot M. 2012, Allons enfants de la patrie: Génération Grande Guerre, Paris: Seuil

Proctor M.T. 2010, Civilians in a world at war, 1914-1918, New York: New York University Press.

Steiner Z. 1977, Britain and the Origins of the First World War, London: Macmillan.

Storr K. 2010, Excluded from the record: Women, refugees and relief, 1914-1929, Oxford: Peter Lang.

Thomas M.G. 2009, Treating the trauma of the Great War: Soldiers, civilians, and psychiatry in France, 1914-1940, Baton Rouge: Louisiana State University Press.

van Bergen L. 2009, Before my helpless sight: Suffering, dying and military medicine on the Western Front, 1914-1918, Farnham: Ashgate.

Watson A. 2009, Enduring the great war: Combat, morale and collapse in the German and British armies, 1914-1918, Cambridge: Cambridge University Press.

Westerhoff C. 2012, Zwangsarbeit im Ersten Weltkrieg. Deutsche Arbeitskräftepolitik im besetzten Polen und Litauen 1914-1918, Paderborn: Ferdinand Schöningh.

\section{Indice delle recensioni in "First World War Studies" ("Fwws") dei testi citati}

on Becker 2010: Lalande J.G. 2012, "Fwws", 1

on Crouthamel 2009: Leese P. 2012, "Fwws", 2

on Gumz 2009: Meaher A. 2011, "Fwws", 1

on Guoqi 2011: Rowe S.E. 2012, "Fwws", 2

on Horne (ed.) 2011: Chickering R. 2011, "Fwws", 1

on Jones 2010: McCrae M. 2012, "Fwws", 2

on Jones, Brien, Schmidt-Supprian (eds.) 2008: Connor J. 2011, "Fwws", 2

on Kaes 2009: Leese P. 2012, "Fwws", 2

on Laskin 2010: DeWitt P. 2011, "Fwws", 1

on Linker 2011: Salvante M. 2013, "Fwws", 2

on Mayerhofer 2010: Knežević J.L. 2013, "Fwws", 1

on Patterson 2008: Vuic K.D. 2011, "Fwws", 2

on Pignot 2012: Hanna M. 2013, "Fwws", 1

on Proctor 2010: Gregory A. 2013, "Fwws", 2

on Storr 2010: Little B. 2011, "Fwws", 1

on Thomas 2009: Keller R.C. 2011, "Fwws", 2

on van Bergen 2009: Meyer J. 2012, "Fwws", 2

on Watson 2009: Madigan E. 2012, "Fwws", 1 


\section{Risorse on line}

International First World War Studies, Bibliography http://www.firstworldwarstudies.org/bibliography.php

Trentino Grande Guerra, Bibliografia $\mathrm{http}: / / \mathrm{www}$. trentinograndeguerra.it/context.jsp?ID_LINK=28\&area=9

Itinerari della Grande Guerra, Bibliografia http://www.itinerarigrandeguerra.it/code/14434/Bibliografia

The Great war 1914-1918, Bibliography http://www.greatwar.co.uk/research/books/bibliography.htm

Wikipedia, Bibliography of World War I http://en.wikipedia.org/wiki/Bibliography_of_World_War_I

Yale University Library Catalog, Bibliography of World War I http://orbexpress.library.yale.edu/vwebv/search? searchType $=3 \&$ searchCode $=\mathrm{SUBJ} \%$ 2B\&searchArg=World+War\%2C+1914-1918+Bibliography\&recCount=50\&dbCode $=\mathrm{LOCAL}$ 